\title{
Digital Sketching Applications for Design of Multicultural Toys Based on Himavanta Creatures from Thai, Laos, and Myanmar Fine Arts
}

\author{
https://doi.org/10.3991/ijim.v14i09.13133 \\ Sittisak Rattanaprapawan $\left.{ }^{\bowtie}\right)$, Apisak Sindhuphak, Krissana Kiddee, \\ Sompol Dumrongsatian \\ King Mongkut's Institute of Technology, Bangkok, Thailand \\ $57603034 \mathrm{kmitl} \cdot \mathrm{ac} \cdot \mathrm{th}$
}

\begin{abstract}
The research focuses on digital sketching aspect through a depiction of Himavanta creatures and characters from Thailand, Laos, and Myanmar, where the details of paintings, sculptures, and architectural features as well as multicultural aspiration and imaginations are considered main variables. The research analyzed Himavanta creatures'cultural identities, sculptural forms and patterns, colors, and characteristic structures and implemented these identities into a design process, which then initiated a digital sketching process to transfer the artwork into designing multicultural toys. The evaluation processes comprised of eight design aspects including 1) concepts and theories of toy design, 2) contents connectedness, 3) discretion to communities, 4) suitability of Himavanta creature, 5) numbers of character sets in basic level, 6) numbers of character sets in experienced level, 7) content management for toy design process, and 8) digitize design procedures. The research results show that the Himavanta creatures including Singha, Chinese lion, Kochasri, Garuda and Swan that based on the digital sketch from paintings, sculptures, architectural features, as well as artisan's imaginations in the multicultural societies were significantly similar.By testing and applying the concepts and theories of multicultural toy design processes from digital sketches, each final design found to be practical and displayed effective cultural means that remains true to its mood and tonality of its cultural interpretation. Consequently, the level of satisfactory evaluation on the process of the design samples, the awareness effectiveness, and the toy's interpretation shows the value of $\bar{x}=4.46$ and the value of 0.58 standard deviation. The satisfaction on the design works shows that awareness and interpretation consistency indicate $\overline{\mathrm{x}}=3.84$ and the standard deviation was at 0.65 .
\end{abstract}

Keywords-Digital Sketching Applications, Toy designs, Multi-Cultures, Himavanta Creatures, and Art and Architectural Content

\section{Introduction}

A toy had a history as old as human civilization itself. Stick, stones, or broken branches served as early form of human's expression to physical tactility, where the 
notion of play serves as reflex to basic emotion. Thus, "play toys" representing ways to pass on the learning through such basic technology, sense of pride, and discovery of physical interaction. Applied technology then through toys, do not only provide entertainment but also encourages learning, strengthens brain complexity, support imagination and benefits to human thinking development [1]. Toys have been designed and developed over the generations [2], resulting in a wide range of changes to keep up with the technological advancement.

In 1990 [3], the Designer Toys, also known as Art Toys, were created by a group of toy lovers called Art Toy artists who hosted a toy exhibition. Ever since art toys are popular throughout the world [4] including in Thailand, where collectibles and novelties created by designers and artists [5] were made from materials such as plastic, cloth, vinyl, wood, metal, resin, or paper, and typically released in limited numbers and without reproduction version. With such techniques as color dyeing matching to digital sketch rendering, the art toys gained a distinctive identity and colorful characteristic, which revealed an art phenomenon generated for a toy industry. These techniques also added additional value for toys [6] and generates new perspectives in owning and increased art and design appreciation to a new level.

As the design emphasis the focus on technologies digital educational environments the experience and creative [7] [8], transforming working styles both hand skills and digital painting into a borderless working manner; working anywhere and anytime, without any restrictions. Only by a smartphone or tablet is sufficient to do the necessary design works [9]. With the use of network and smart mobile technologies, it allows us to monitor and coordinate across different locations and broadens more channels of developing as well as learning. Therefore, a design process and technology implementation greatly help to support users on the demanding needs in translating the detail of character to a new form of technological representation. Applications for adaptive and flexible learning allows the users to exploit self-learning using available technologies [10] to help in sketching and drawing with more accuracy and with creative outcome. They are like a device used for virtual design or alternative ways to art works' creation.

\subsection{Toy design}

The concept of toy design must be simple and clear using the profound and natural geometric patterns [11]. Meanwhile, the toy design framework consists of culture and economy [12] that focuses on using design expression as a tool to present the relationship between arts and behaviors -driving the toys to act as a symbolic communication with other means of social utility and connectivity. This is likewise in discussing about the cultural design frameworks for creating toys to connect the world of adults and the world of children [13] where the design of multicultural toys has a common goal of internationalization awareness that assist children to gain the social skill to associate and interact through playing and learning, while the adults emphasis on the aspect of hobby and form of leisure activities. 


\subsection{Digital sketching}

Digital Sketching is a powerful tool that supports artwork and design creation, suitable in the digital age that accommodates agility, speed and precision. Sketching is the easiest step to convey ideas into a concept. It is a visualizing process, a way of recording, or displaying signs to remind ideas, which can either start with something that already exists or start over with the new idea. Designers use digital sketching application as a tool to transfer ideas, leading to results and application to justify certain area and process [14] that helps accentuate the art by the hands and further.

The application' capabilities include powerful drawing tools such as line blur, weight and line brush size setting, color control, and vision management. These digital features are also accurate in creating both $2 \mathrm{D}$ and $3 \mathrm{D}$ imagery that focus on expressive notion of color and pattern discovered from the mythical figures that allowed alternative perspectives to the visual form, composition, and design paradigm.

\subsection{Multicultural design}

All human being has their own way of life, thoughts, feelings, and expression that have arisen from learning throughout their lives. There are three principles important in culture that must be considered for multicultural design:

1. Symbolic expressions,

2. Ancestors or heroes

3. Beliefs and religions.

These three beliefs will lead to the design with strong profound cultures or social representation. The use of digital tools for this research in enhancing the quality inspires the transfer of abstract symbols and patterns into simple and distinct digital forms. It can also solve the problem of transforming the numerous layers of structures into an impressive sketch [15], ready for design use or contents distribution.

\subsection{Himavanta}

Himavanta is a forest in literature and in the Trailokya (meaning: Three Worlds or Realms), belief according to Buddhism and Hindu. It is a representation that connects the Himavanta forest to an objective place, which mentioned its location on the Himavanta or Himalayas mountain. The researcher is interested in presenting Himavanta creatures as a case study to scope into methods of ways to inspire contemporary development to initiate an approach to collaborate with their thoughts on the matter.

However, there are only few studies on the design of multicultural art toys and linkage between personal thoughts and social opinion, understanding and imagination in regard to Himavanta creatures' characters in Buddhist multicultural societies of Southeast Asia [16]. Few researchers have ever studied and compared such issues to design and produce the art toys through the digital sketching/digital painting applications or for furthering $3 \mathrm{D}$ printed prototype. 
From such contents, this research intents to study the Himavanta creatures as fine arts appearing from the paintings, sculptures and architectures features based on cultural beliefs and imaginations from people in Buddhist multicultural societies. With the use of digital sketching application, the result may also yield possibilities of the art toy designs as a testament to Multicultural accentuation from Thai design.

\section{Material and Experimental Procedure}

This research comprised of both qualitative and quantitative methods. By selecting 3 Buddhist countries in ASEAN community consist of Thailand, the Lao People's Democratic Republic, and the Republic of the Union of Myanmar as a case study, of which each capitals city of Bangkok, Vientiane, and Rangoon were chosen for their cultural merit representation (six temples per city with the total of 18 temples). Each countries' architectural buildings and structure features such as important chapels and temples as well as their sculptures, paintings, and Buddhist literatures were studied. The procedures are as the following:

1. Conducting document surveys with search and discovery on knowledge regarding Himavanta, where comparative and contrast method of the three cultural identities derived on visual forms, personalities, patterns, colors, and structures of mythical creatures were collected. The obtained data was synthesized and analyzed for future used as a design guideline as demonstrated from figure 1, that several shared perspectives on creatures' characteristic might be different, however the core identity and the inner meaning of the character has shown connection of relationship of art, contemporary features, and style from the three countries. That conclude to pertain 4 popular culture influential factors that drive the interest of similarities and differences between the three countries.

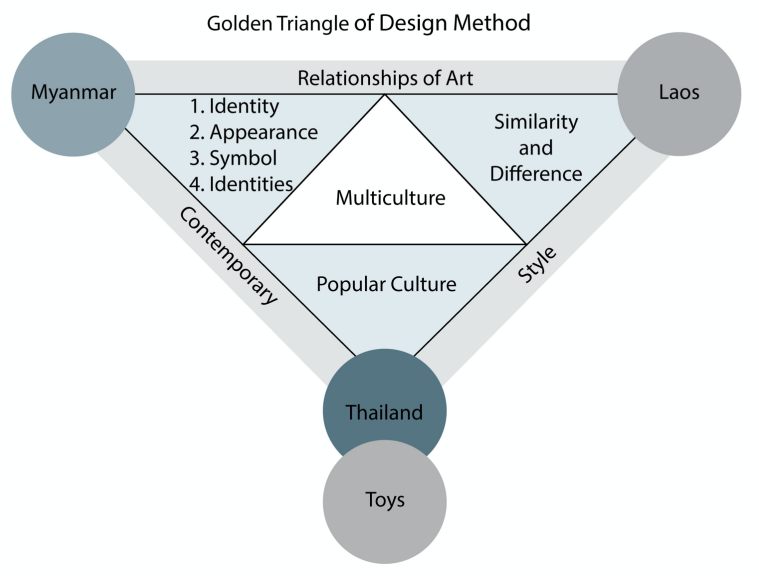

Fig. 1. Golden Triangle of Design Method, demonstrated the connection of three cultural mythical characters to the core of Multi-culture and Popular Culture. (Grounded from the lecture series at KMITL, (2016). 
Furthermore, the collective data from the three mythical selections displayed from figure 2 shown the studying of the multi-culture representation, which shared the common characteristics of perception through thoughts and symbols derived from the Culture, belief or religion creation. For example, "Singha" can always be perceived as "Singha" because of its structure and gestures no matter where they appear despite the differences in decorative patterns and techniques that artisans elaborately conveyed the uniqueness and identity of the character's societal respect and positioning as mythical creature of all three cultural points of view.

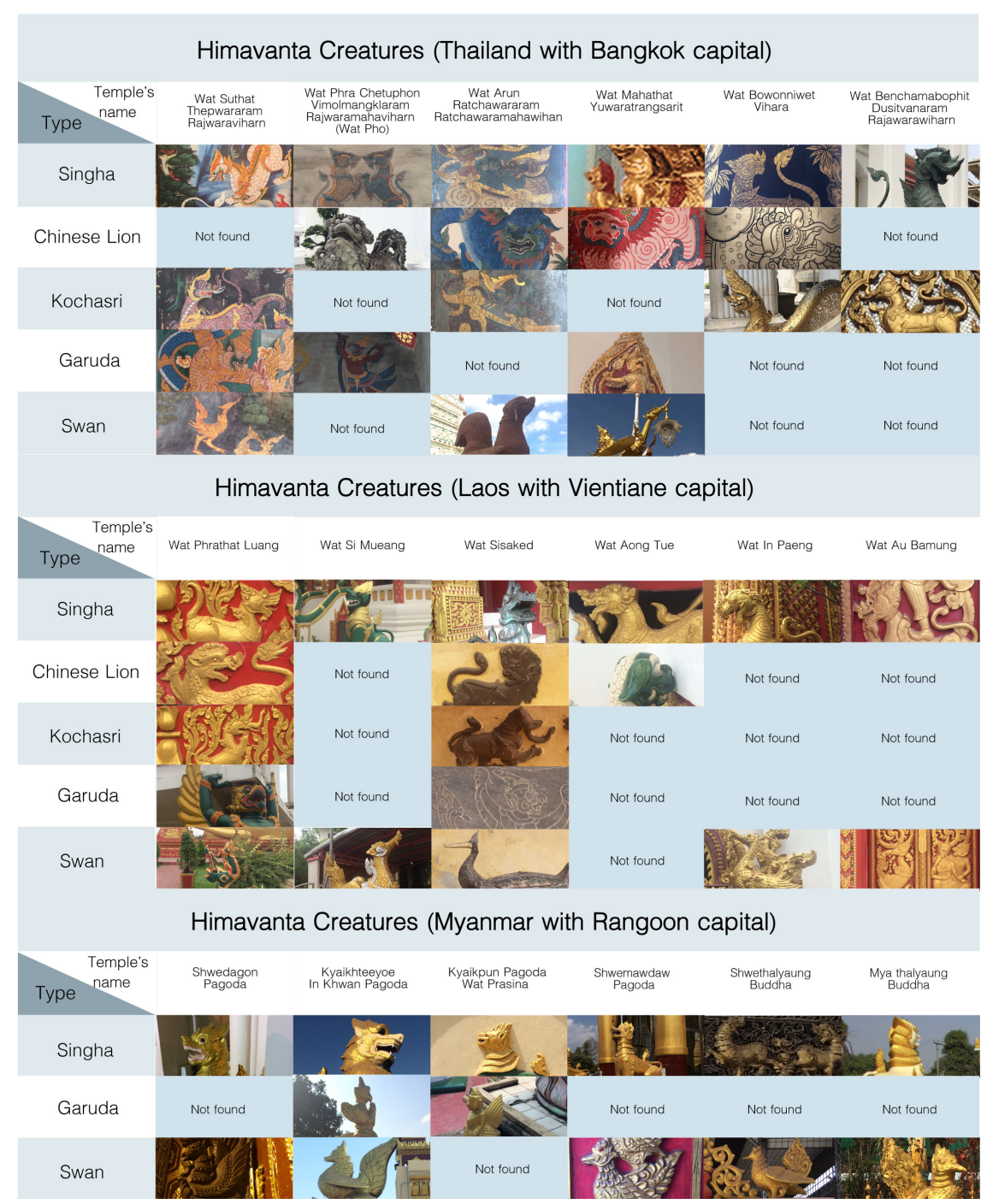

Fig. 2. Matrix Chart of data and details for Himavanta creatures in 3 countries including 1) Thailand with Bangkok as the capital, 2) Laos with Vientiane as the capital, and 3) Myanmar with Rangoon as the capital. 
After analyzing the chart, the researcher summarized as follows: there were 5 types of Himavanta creatures including Singha, Chinese Lion, Kotchasri, Garuda, and Swan in Thailand and Laos. Of all five types, creatures found through Laos was influenced by Thai Arts. Whereas, the Chinese Lion and Kotchasri were not found in Myanmar due to religious belief of using Himavanta creatures that held different beliefs and usages.

Main ideas for multicultural toy design were carried out by analyzing the classification, decorative patterns, colors, and common structures of such Himavanta creatures. There was also an analysis on the research data and the design process using descriptive statistical methods such as interquartile range (IR)to justify the correlation between the changes and acceptances from each culture practices and beliefs.

2. The sample size was determined for the research using Krejcie and Morgan tables to estimate a proportion of population and determine Himavanta creatures' structures, patterns, and proportions. A stratified random sampling and a result evaluation on various elements were undertaken to gain a design process of multicultural toys as shown in figure 3 , which emphasize on the transitional idea through the process that encompassed the combination of the three cultural approaches to the Himavanta mythical meanings. The sample group of 19 expertise assist in determining the validity and suitability satisfaction, where 5 artists and 181 students were asked to test the 18 area of satisfactions.

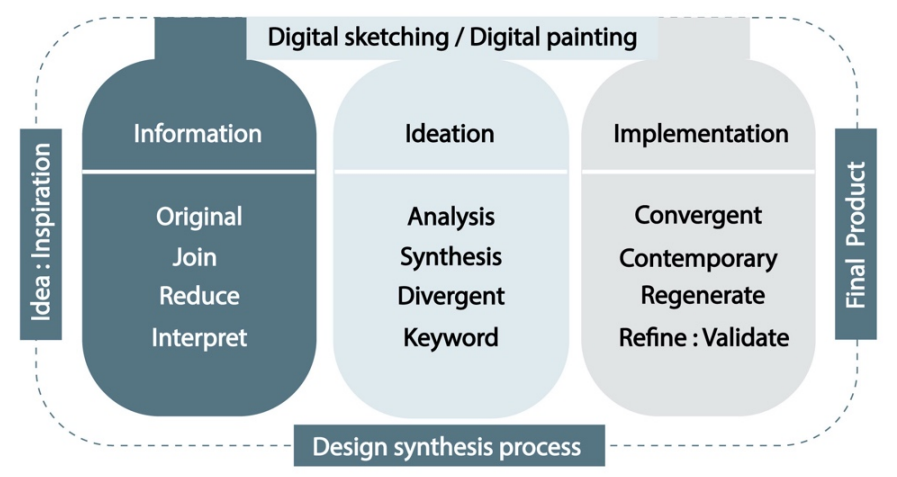

Fig. 3. An analysis of a design process for multicultural toys, which utilize digital sketching and painting into the process circulation.

3. The design process was tested using cognitive styles while the studied principles of multicultural toys were experimented by mean of Delphi method, for which to relate the result of forecasting the process that can analyze data and measure results leading to conclusions. In this method, experts in cultures and fine arts, toy designers, character designers, and art toy artists provided their opinions through design work creation using the digital sketching application to interpret line-weight, details, and visual form of the characters, all based on the eight satisfaction factors in regard to the design interpretation. 


\section{Results and Discussion}

The study concerned with Singha, Chinese lion, Kochasri, Garuda and Swan appearing as Himavanta creatures demonstrated through figure 4 comparative chart, allows the collective finding to be viewed as a source of art impression that can traced the meaning and intention from traditional methods of storytelling. Important Buddhist temple and architectural features, sculptures, paintings [17] and Buddhist literatures as well as in imaginations of people in the Buddhist multicultural societies from Thailand, the Lao People's Democratic Republic [18] and the Republic of the Union of Myanmar [19] are shown in figure 4. By justify the significant of the proper implication of the digital sketch, where such tools help in preserving the artistic rarity of form and lineweight is a mission of paying respect to honor the historical past through existing technology.

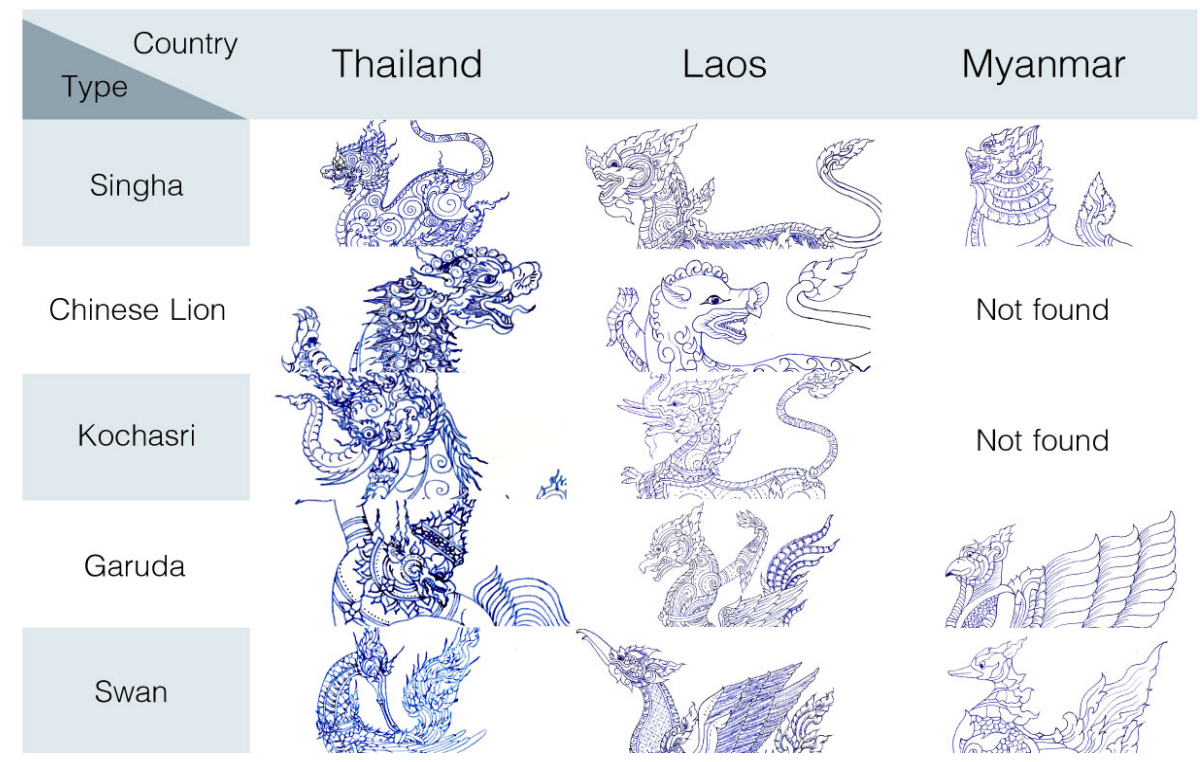

Fig. 4. Comparison of Himavanta Creatures between the three Buddhist Countries in ASEAN Community (Sketches and drawings made by hands for data collection).

As figure 4 displays, the area survey found various sketching methods when creating a linear system from duplicate patterns of 2 or more Himavanta creatures. The sources of the pattern can be described as follows: the prototypes from the sculpture, painting, carving, and decorative items that appear in the architecture are meticulous, subtle, deferential but situated as hidden features. Of which, visual form and pattern can be recognized as similar in form and composition when related back to Thai' Himavanta creature. As figure 5 will yield, the point of similarity stresses the insignificant of visually differences where now the prominent details and patterns emphasize on the connection of composition and visual form that people can relate and starting to make connection to the digital world. 


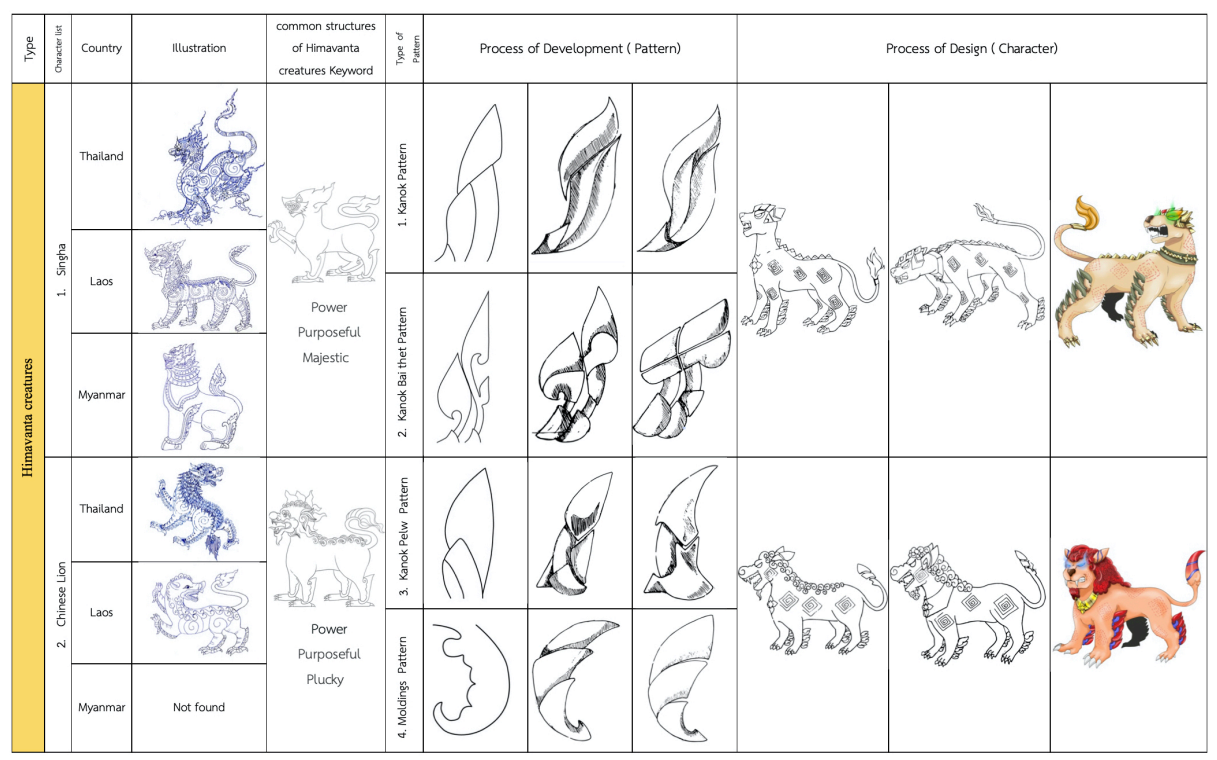

Fig. 5. Procedures of using conceptual models and designing principles of multicultural social toys through design work synthesis and color and form analysis.

By showing the procedures of using conceptual models and designing principles of multicultural social toys, 2 contrasting designs appeared to hold characters trait that are connected. The development system can be classified into 2 characteristics: character structure and decorative pattern on the character. This is the sequential development process in which the designer must add detailed patterns in the line drawings, coloring in $2 \mathrm{D}$ work and adding shadows to create a $3 \mathrm{D}$ atmosphere respectively. In table 1 , demonstration of transferring the main idea to coincide with possible adjustment with digital applications. 
Table 1. Categorization of usability criticism and potential for character development.

\begin{tabular}{|c|c|c|}
\hline Usability Criticism & Method Models & Samples \\
\hline \multicolumn{3}{|l|}{$\begin{array}{l}\text { The development of a common struc- } \\
\text { ture of the Himavanta creature's key- } \\
\text { word/ main idea through the character } \\
\text { can adjust gestures to feel the various } \\
\text { mood such as walking or sitting. }\end{array}$} \\
\hline \multicolumn{3}{|l|}{$\begin{array}{l}\text { Himavanta creatures' patterns with a } \\
\text { different gesture can adds decorative } \\
\text { patterns to fulfill additional appear- } \\
\text { ance } \\
\text { and mood. }\end{array}$} \\
\hline $\begin{array}{l}\text { The character's process of shading } \\
\text { tone and form coloration can fulfill } \\
\text { the overall gestures and emotional } \\
\text { perception. Therefore, familiarity of } \\
\text { traditional details helps to develop and } \\
\text { design a character with more accu- } \\
\text { racy. Additional detail from this step } \\
\text { is adding individual imagination and } \\
\text { insert other elements, of which base } \\
\text { on the Himavanta creature core and } \\
\text { can combine to other imaginative as- } \\
\text { pect in the future. }\end{array}$ & dow & \\
\hline & Color & \\
\hline
\end{tabular}

From table 2, the evaluation of cognitive styles and principles of multicultural toy design using Delphi method by the experts in cultures and fine arts, the toy designers, the character designers and the art toy artists. By analyzing the sample groups or the statistical value using the descriptive and inferential statistics, interquartile range (IR) are in good significance level. This represents possibilities to design multicultural toys using Singha, Chinese lion, Kochasri, Garuda and Swan as a model. 
Table 2. An evaluation of cognitive styles and principles of multicultural toy design $(\mathrm{N}=19)$

\begin{tabular}{|l|c|c|l|}
\hline \multicolumn{1}{|c|}{ Evaluation } & Mdn & IR & \multicolumn{1}{c|}{ Interpretation } \\
\hline Study Process and Toy Design Process & 5.00 & 1 & Very consistent \\
\hline Information Accuracy & 5.00 & 0.75 & Most consistent \\
\hline Suitability of Data Storage Location & 5.00 & 0 & Most consistent \\
\hline Suitability of Himavanta Creature Sets & 5.00 & 1 & Very consistent \\
\hline Numbers of Character Sets in Basic Level & 5.00 & 1 & Very consistent \\
\hline Numbers of Character Sets in Experienced Level & 5.00 & 1 & Very consistent \\
\hline Database Management for Design Process & 5.00 & 0.88 & Most consistent \\
\hline Total & 5.00 & 0.80 & Most consistent \\
\hline
\end{tabular}

As seen from Table 2, the experts have the opinion that all procedures of using conceptual models and designing principles of multicultural social toys in the step of design work development are totally consistent. The details are as follow: 1 . Study Process and Toy Design Process ( $M d n=5.00, I R=1,2$. Information Accuracy $(M d n=5.00, I R=0.75)$, 3. Suitability of Data Storage Location $(\mathrm{Mdn}=5.00, \mathrm{IR}=0)$, 4. Suitability of Himavanta Creature Sets $(M d n=5.00, I R=1)$, 5. Numbers of Character Sets in Basic Level $(\mathrm{Mdn}=5.00, \mathrm{IR}=1), 6$. Numbers of Character Sets in Experienced Level $(\mathrm{Mdn}=5.00$, $\mathrm{IR}=1$ ), and 7. Database Management for Design Process ( $M d n=5.00, \mathrm{IR}=0.88$ ).

The design results were used to evaluate the eight aspects of satisfaction as detailed in Table 3. With an analysis of the cognitive styles and studied methods of multicultural toy design, the statistical results show that the mean $(\overline{\mathrm{x}})$ and the standard deviation (SD) are in good significance level, where the data will be considered as the design concept indicates through the visual form and line stroke. When interpreted the statistical result, it indicates that all experts strongly agree to use the guidelines and methods studied in this research in order to perceive the authenticity of the traditional intention in helping to design for the future multicultural toy.

Table 3. A result of satisfaction evaluation toward the study and design process of Himavanta creatures $(\mathrm{N}=5)$

\begin{tabular}{|l|c|c|l|}
\hline \multicolumn{1}{|c|}{ Evaluation Measurement } & $\overline{\mathbf{x}}$ & SD & Interpretation \\
\hline Concepts and theories of toy design & 4.50 & 0.50 & Most consistent \\
\hline Contents connectedness & 4.18 & 0.57 & Very consistent \\
\hline Discretion to communities & 4.43 & 0.83 & Very consistent \\
\hline Suitability of Himavanta creature & 4.40 & 0.55 & Very consistent \\
\hline Numbers of character sets in basic level & 4.60 & 0.55 & Most consistent \\
\hline Numbers of character sets in experienced level & 4.60 & 0.55 & Most consistent \\
\hline Content management for toy design process & 4.47 & 0.54 & Very consistent \\
\hline Digitize design procedures & 4.51 & 0.52 & Most consistent \\
\hline Total & 4.46 & 0.58 & Very consistent \\
\hline
\end{tabular}

According to the table 3 , it is found that a result of satisfaction evaluation toward the study and design process of Himavanta is all consistent. The details are as follow: 1 . Concepts and theories of toy design $(\overline{\mathrm{x}}=4.50, \mathrm{SD}=0.50), 2$. $\mathrm{v}(\overline{\mathrm{x}}=4.18, \mathrm{SD}=0.57), 3$. 
Discretion to communities $(\overline{\mathrm{x}}=4.43, \mathrm{SD}=0.83), 4$. Suitability of Himavanta creature $(\overline{\mathrm{x}}$ $=4.40, \mathrm{SD}=0.55), 5$. Numbers of character sets in basic level $(\overline{\mathrm{x}}=4.60, \mathrm{SD}=0.55), 6$. Numbers of character sets in experienced level $(\overline{\mathrm{x}}=4.60, \mathrm{SD}=0.55)$, 7. Content management for toy design process $(\overline{\mathrm{x}}=4.47, \mathrm{SD}=0.54)$, and 8 . Digitize design procedures $(\overline{\mathrm{x}}=4.51, \mathrm{SD}=0.52)$.

As the notion of digital mobile learning leads to a better resource's organization and adaptability, accessibility to work anywhere, anytime and transportability of mobile platforms [9] can mostly benefits the design process. As application for designers serves as a tool supporting sketching and drawing documentation, a feature that allows the collection of visual forms and line strokes to transfer onto various platform for design purposes can serve as having a portable laboratory [20]. They reduce costs of materials and equipment for creating unnecessary repetitive design or art works, lower operation time, and provide a variety of specific tools to complete the job more effectively. With sophisticated capabilities in sketching, drawing, painting, and building realistic textures, these applications are becoming the ideal tools to initiate as well as to challenge designing and drawing procedure and process of the past [21]. Thus, generated the use of new applications for designers to control and expand the creativity as a design studio simulation, which offer ways to apply technologies and features to support creating works from imaginations with mobile devices system created to use on any locations [22]. Various collective data as seen from table 4, shows the available digital applications and tools use in today mobile creative field.

Table 4. Ten applications for digital sketching / digital painting.

\begin{tabular}{|l|l|l|l|}
\hline Icon & \multicolumn{1}{|c|}{ Apps } & \multicolumn{1}{c|}{ Skills } & Platforms \\
\hline SketchBook & Sketching + Painting & $\begin{array}{l}\text { iPad, Tablet, } \\
\text { Smartphone }\end{array}$ \\
\hline & Assembly & Sketching + Painting & iPad, Smartphone \\
\hline & Inspire & Sketching + Painting & iPad, Smartphone \\
\hline & Tayasui Sketches & Sketching + Painting & iPad, Smartphone \\
\hline
\end{tabular}




\begin{tabular}{|l|l|l|l|}
\hline \multicolumn{1}{|c|}{ Apps } & \multicolumn{1}{|c|}{ Skills } & Platforms \\
\hline Icon & Zen Brush 2 & Sketching + Painting & $\begin{array}{l}\text { iPad, Tablet, } \\
\text { Smartphone }\end{array}$ \\
\hline & Adobe Illustrator Draw & Sketching + Painting & iPad, Smartphone \\
\hline & ASKetch & Sketching + Painting & iPad, Smartphone \\
\hline & Paper by FiftyThree & Sketching + Painting & iPad, Smartphone \\
\hline
\end{tabular}

According to the 10 cited applications [23], they are all available on different platforms. There are tools that come with over 190 brushes with choices of digital brush and customization features. Thus, performances showed the congruency in virtual space and provide the same effect as working on real paper, and helps maintain the sketching strokes and line-weight smoothly and naturally. In this experiment on Himavanta creatures' creation, the researchers randomly select SketchBook application out of all above examples to be used as a tool by the sample group (designers), with reasons that the application help expressed visual forms and patterns by transferring the close accuracy of line-weight recorded by traditional hands sketch.

The selection was in accordance with the quality control principles of data triangulation, which consists of controlling the operation of people, devices, places, equal working hours under the same problem. The designers were assigned to draw one Himavanta creatures out of Singha, Chinese lion, Kodchasri, Garuda, and Swan.

Autodesk SketchBook app contains a variety of essential drawing tools such as perspective guides, fill, effects sets for working with layers, as well as capabilities to customize paint brush sets, layer systems or color selection tools, which perceived to have natural feel of traditional tools and designed with comfortability of use when designing variation of Himavanta shapes and characters detail features. 


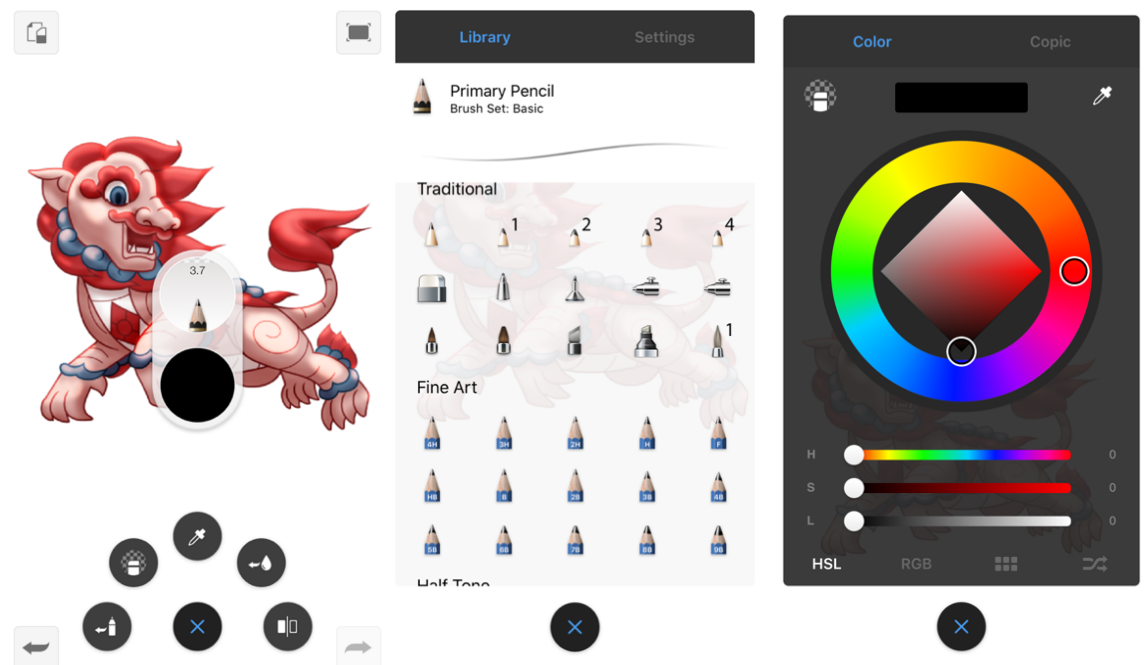

Fig. 6. The design through SketchBook application with specific customizable tools

Furthermore, the application is a tool to facilitate work creation through a variety of devices with touch screen systems such as platform of mobile phones, tablets, and iPads. It transcends various limitations and can be used anywhere. It is economical, modern, fast, and can save into many file extensions such as JPG, PNG, vector, TIF, GIF, Ai, and PDF. This is considered a technological advancement supporting the creature creation in the digital era.

Therefore, the researcher would like to use the applications as a design learning platform and a reference for design supports. With these apps, users can study independently and access anytime even when they are outside the enclosed classroom. These applications can be used in product developments to help general people understand technologies and solve problems in the future [24]. In addition, the applications have been continuously developed and adapted to respond with users and to support in working on ideas of anywhere and anytime. These digital sketching/digital painting applications are built to meet creators need. Advantages of using a smartphone or tablet are mobility and portability to easy access of wireless communication to share data in real time [25] and link through platforms. The applications are similar to a pocket-sized computing device with a display screen that functions by touch input or a small keyboard [26]. Smartphones and tablets are the popular choices when people need supports and convenience. In general, these devices can provide almost everything necessary for the design processes [27]. Eventually, working through the applications can provide user's opportunities to explore their personal potential rather than for the learning purposes [28]. It also strengthens interaction between humans and portable devices [22] that link to the practices that technology provide solution when approach the problem with human interaction in mind.

In figure 7, 2D art toy designs based on cultures and fine arts by toy designers, the character designers, and the art toy artists (experts) have applied their design suggestion 
by using digital sketching/digital painting applications. These creations applied the cognitive styles and toy design methods into this research that revealed the interpretation of cultural arts and modified architectural features into eventually the multicultural toy designs idea as both 2 and 3 dimensional solutions.
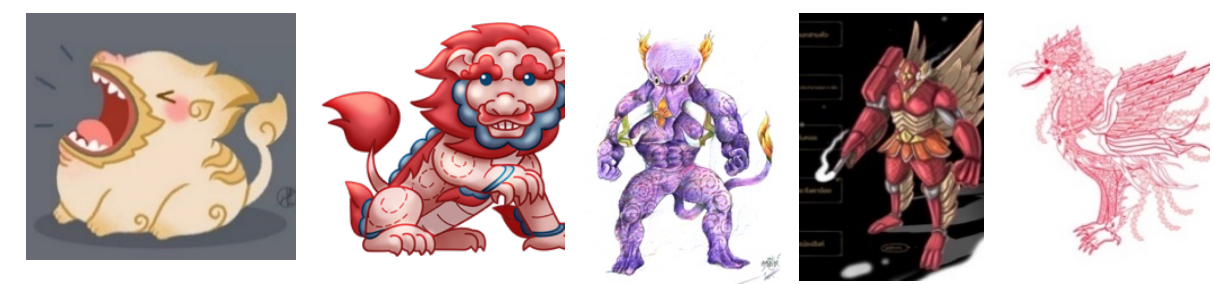

Singha

Chinese Lion

Kochasri

Garuda

Swan

In term of design selection, as shown in the table 5, the result of satisfaction evaluation on the 2D works of Himavanta creature toys - Singha, Chinese lion, Kochasri, Garuda, and Swan, responded by the experts represents the statistical analysis as a good significance level of mean $(\overline{\mathrm{x}})$ and standard deviation (SD). When interpreted the statistical results, the five characters show the high possibility to convey the Himavanta creatures' contents have trait of a contemporary concept, which combined both conventional drawings and digital sketches resulting toward the methods and strategies for later use when designing multicultural toy processes.

Table 5. An evaluation on the design works of Himavanta creatures as toys $(n=181)$

\begin{tabular}{|l|c|c|l|}
\hline \multicolumn{1}{|c|}{ Evaluation on Design Works } & $\overline{\mathbf{x}}$ & SD & \multicolumn{1}{c|}{ Interpretation } \\
\hline Singha type & 3.60 & 0.56 & Very consistent \\
\hline Chinese Lion type & 3.75 & 0.65 & Very consistent \\
\hline Kochasri type & 3.77 & 0.70 & Very consistent \\
\hline Garuda type & 4.23 & 0.70 & Very consistent \\
\hline Swan type & 3.86 & 0.62 & Very consistent \\
\hline
\end{tabular}

From the table 5 , it is found that an evaluation on the design works of Himavanta creatures as toys is all consistent. The details are as follows: 1 . Singha type $(\overline{\mathrm{x}}=3.60$, $\mathrm{SD}=0.56), 2$. Chinese Lion type $(\overline{\mathrm{x}}=3.75, \mathrm{SD}=0.65), 3$. Kochasri type $(\overline{\mathrm{x}}=3.77$, $\mathrm{SD}=0.70), 4$. Garuda type $(\overline{\mathrm{x}}=4.23, \mathrm{SD}=0.70)$, and 5. Swan type $(\overline{\mathrm{x}}=3.86, \mathrm{SD}=0.62)$. Deriving the 181-sample group for the research, information age, digital sketching and mobile learning is gaining popularity and provides learning privacy [29]. Smartphones and tablet are ideal for supporting learning and maintaining motivation for the new generation. It also allows users to share and edit information regardless of the time and location [30]. These devices are favorable choices for those who need the assistance and convenience superior to a conventional computer, in situations where they are not practical to carry [27]. Using a digital sketching application after data survey, an application called Autodesk SketchBook app, has shown strong recommendation from the experts where the design works by such application is effective and offer features and 
tools that maintain the artistic expression as if drawn by hands. The patterns of Himavanta creatures where Garuda selected as exemplar character due to selective profiling was drawn from first, left and right profiles, to front side, and with the back side to create a model using Zbrush render. This combination of both programs, where notion of digital sketches and a digital sculpting tool combines $2 \mathrm{D} / 3 \mathrm{D}$ modeling, texturing and painting that becomes an objective form, can generated a tangible solution from the complex conceptual idea.

In this case, Zbrush uses a "pixel" technology which stores lighting, color, material, and measurable depth information for all objects on the screen. SketchBook App as well hold similar capability in creating "high-resolution" images and models as Zbrush, where both has dynamic levels when adjusting resolution, allowing creators artistic freedom to changes models to reflex cultural interpretation [31]. Consequently, the end process that obtained the designed model can be prepared for applying the digital data into a $3 \mathrm{D}$ rendering or production of printed prototype.

\section{Conclusion}

This research shows that there is significantly similarity among Himavanta creatures-Singha, Chinese lions, Kochasri, Garuda, and Swan in Southeast Asia countries including Thailand, the Lao People's Democratic Republic, and the Republic of the Union of Myanmar based on their architectural features within buildings and places such as important Buddhist chapels and temples as well as their sculptures, wall paintings and Buddhist literatures. By experimenting and applying the concepts and theories of multicultural toy design studied in this research into the art toy design through the digital sketching and model building application called Autodesk SketchBook app and Zbrush respectively, the obtained statistical analysis demonstrates that this is a practical approach to conduct design concept through procedures from art transferred knowledge by using digital tools on the device and the existing network that is readily available as part of everyday real situation.

This research studied the multiculturalism theories, which apply the testing group via art interpretation from mythical figures and the application of cultural signs in design works [32] to finally conclude into the designing of character by designing a new idea of learning from conventional sketching and at the same time appreciating the meaning of cultural arts through mobile devices and available applications from today virtual market. It also did cross-discipline studies from various fields including history, literature, religion, society, education, psychology, behavior, and technology, thus solidify an interdisciplinary study, which is instrumental in the creation of universal things and common phenomena arising from many dimensions [33] in today society.

This research is based on beliefs, faith and imagination as the core, and reflects on the environment affecting the perception of Himavanta characters. Systematically creative process, which is very smart and beneficial, perhaps guided to obtain clear solution, where the thought system yielded a good design work with quality despite the different experience of each designers. Reflecting the differences of just solely uphold traditional drawing method, then to currently allow the idea of crossed method, new 
practices can perhaps be seen as way to regenerate learning process and not just ways to appreciate the traditional arts -and not having ways to express nor response to the core art knowledge for contemporary daily life.

The designer demonstrates creativity using cognitive styles and product design principles for multicultural toys. The Garuda or Singha design reflects individual experiences and thoughts. With the programs, which simplifies the design steps, the quality level of character's detail and visual form are ready to apply 2D works into 3D prototype. On an important note, in order to gain a cultural design acceptancy for the cultural merits and pertaining an international standard, a knowledge base creation must be recognized. Thus, establishing a workshop helping the future learning of art process can benefits in areas such as cross-combination of character's minor and dominant details. The new appearances or innovative form can then occur from such process. Designing methods may have a resulting possibility to be used for character creation and art toy, however thinking and visualization process as the amalgamation bridges between the past and present outcomes, with a mixture of cultural future; may now finally be revealed.

\section{$5 \quad$ References}

[1] Cole, A. J., \& Brooks, E. P. (2017). Toy Story: Childhood versus Children in Toy Museums. Museum and Society, 14(2), 294-312. https://doi.org/10.29311/mas.v14i2.675

[2] Putnark, V. (2018). Toys are not small matters when it comes to learning to become adult. The Matter Social. Retrieved from https://tinyurl.com/s7amyzo

[3] Akkpark, Y. (2019). Art Toy so what is it. Retrieved from https://tinyurl.com/wmds753

[4] Bou, L. (2014). We are indie toys, make your own resin characters. Harper Collins. New York, United States.

[5] Jeremy, V. E. (2004). Vinyl Will Kill.Gingko Press. Hong Kong, Hong Kong. pp. 5-6

[6] Coelho, D. A., \& Fernandes, S. A. (2013). Toy Design: A Methodological Perspective. The International Journal of Designed Objects, 7(1), 51-64. https://doi.org/10.18848/2325$1379 / \mathrm{cgp} / \mathrm{v} 07 \mathrm{i01} / 38675$

[7] Vidakis, N., Barianos, A., Trampas, A., Papadakis, S., Kalogiannakis, M., \& Vassilakis, K. (2019). Generating Education in-Game Data: The Case of an Ancient Theatre Serious Game. Proceedings of the 11th International Conference on Computer Supported Education. https://doi.org/10.5220/0007810800360043

[8] Papadakis, S. (2020). Evaluating a game-development approach to teach introductory programming concepts in Secondary Education. International Journal of Technology Enhanced Learning, 1(1), 1. https://doi.org/10.1504/ijtel.2020.106282

[9] Corrienna, A. T., Hassan, A., Adi, M. A. M., Kang, H. S., Igor, N, \& Marlina, A. (2019). Sakai: A Mobile Learning Platform. International Journal of Interactive Mobile Technologies, 13(11), 96-107. https://doi.org/10.3991\%2Fijim.v13i11.10800

[10] Alaeeddine, Y., Mohammed, B., Ahmed, T, \& Driss, C. (2018). A Cross-Platform Mobile Application for Learning Programming Basics. International Journal of Interactive Mobile Technologies, 12(7), 139-150. https://doi.org/10.3991\%2Fijim.v12i7.9442

[11] Yong-Jin Liu, Cui-Xia Ma, \& Dong-Liang Zhang. (2011). EasyToy: Plush Toy Design Using Editable Sketching Curves. IEEE Computer Graphics and Applications, 31(2), 49-57. https://doi.org/10.1109/mcg.2009.147 
[12] Ginoulhiac, M. (2013). Design Education Through Toy Design. Old and new Paradigms in Architectural Toys Design. Article in International Conference Proceedings Book. pp.1-12 Retrieved from https://tinyurl.com/tgnvgdu

[13] Kudrowitz, B. (2014). Emerging Technology and Toy Design. Retrieved from https://tinyurl.com $/$ sjn 7 bnf

[14] Koutamanis, A. (n.d.). Sketching with Digital Pen and Paper. Computer Aided Architectural Design Futures 2005, 321-330. https://doi.org/10.1007/1-4020-3698-1 30

[15] Mohammad, R., \& Mariano, R. (2005). The Influence of the

[16] Designers' own Culture on the Design Aspects of Products. EAD06 European Academy of Design International Conference. Retrieved from https://tinyurl.com/r52t281

[17] Nakapan, W.,Ku, Y., \& Pattanasirimongkol, A. (2017). The Mathematical Logic Behind Lai Thai a Geometric and Parametric Analysis of the Traditional Thai Pattern. Proceedings of the 22nd International Conference of the Association for Computer-Aided Architectural Design Research in Asia (CAADRIA) 2017, 241-251. Hong Kong. Retrieved from https://tinyurl.com/sqp9exo

[18] [Chareonla, C. (n.d.). Buddhist Arts of Thailand. Buddha Dharma Education Association Inc. Retrieved from https://tnyurl.com/tuw8edx $\% 20$

[19] Sregongsang, S. (2010). A Study of Thailand and Laos Relations Through the Perspective of the Vientiane Sisaket Temple and the Rattanakosin Emerald Buddha Temple. A Thesis Submitted in Partial Fulfillment of the Requirements for the Degree Doctor of Philosophy Program of Architectural Heritage Management and Tourism (International Program) Graduate School Silpakorn University. Retrieved from https://doi.org/10.17501/icoht. $\underline{2016.4110}$

[20] Rattanaprapawan, S., Sindhuphak, A., \& Kiddee, K. (2017). The Symbolic Arts in Buddhist Literatures on the Himmapan Creatures in Multi-cultures Case studies of Thailand, Myanmar, Laos. Proceeding of the 15th DRLE International Conference on Developing Real-Life Learning Experiences: Smart Education for Sustainable Development, June 16 2017, Bangkok, Thailand. pp. AED07-1- AED07-4

[21] Landay, J. A., \& Myers, B. A. (1994). Interactive Sketching for the Early Stages of User Interface Design. https://doi.org/10.21236/ada285339

[22] Yuktirat, C., Sindhuphak,A., \& Kiddee, K. (2018). M-learning for the Art of Drawing: Informal Learning for a Digital Age. International Journal of Interactive Mobile Technologies, 12(5), 152-165. https://doi.org/10.3991/ijim.v12i5.9207

[23] Kwon, E., Camba, J. D., \& Kimbrough, M. (2018). Conceptual Product Design in Digital and Traditional Sketching Environments: A Comparative Exploratory Study. J. of Design Research, 16(2), 131. https://doi.org/10.1504/jdr.2018.092810

[24] Wasuthanasub, T. (2018). 10 An Application that We Think is Ultimately Good for Digital Art Fan. Retrieved from https://tinyurl.com/qvyuaxc

[25] Shah,A., Suhailiezana , \& Che, G. C. K. (2019).Effectiveness of M-Learning Applications for Design and Technology Subject. International Journal of Interactive Mobile Technologies, 13(10), 120-132. https://doi.org/10.3991/ijim.v13i10.11324

[26] Cruz-Flores, R., \& López-Morteo, G. (2010). A Framework for Educational Collaborative Activities Based on Mobile Devices A Support to the Instructional Design. International Journal of Interactive Mobile Technologies, 3(4), 11-17. https://doi.org/10.3991/ ijim.v4i3.1268

[27] Wei, L. (2018). Design of a Digital Art Teaching Platform Based on Automatic Recording Technology. International Journal of Emerging Technologies in Learning, 13(8), 185-189. https://doi.org/10.3991\%2Fijet.v13i08.9050 
[28] Dothang, T. (2014). How to Design a Mobile Application to Enhance Teaching and Learning? International Journal of Emerging Technologies in Learning, 9(3), 4-10. https://doi.org/10.3991\%2Fijet.v9i3.3507

[29] Sun, H. (2017). Design of Education Application based on Shiva 3D Platform. International Journal of Emerging Technologies in Learning, 12(4), 200-206. https://doi.org/10.3991\%2Fijet.v12i04.6685

[30] Yuan, Z, \& Lu, Z. (2019). College English Teaching Status and Individualized Teaching Design in The Context of Mobile Learning. International Journal of Emerging Technologies in Learning, 14(12), 94. https://doi.org/10.3991/ijet.v14i12.10704

[31] György, M. (2013). New Learning Spaces? M-Learning's, in Particular the iPad's Potentials in Education. International Journal of Interactive Mobile Technologies, 1(7), 56-60. Retrieved from https://tinyurl.com/u6jg8bk

[32] Tuengsantia, K. (2015). Software for 3d designing. Retrieved from https://tinyurl.com/w4vhh45\%20

[33] Lin, R., Sun, M.-X., Chang, Y.-P., Chan, Y.-C., Hsieh, Y.-C., \& Huang, Y.-C. (2007). Designing "Culture" into Modern Product: A Case Study of Cultural Product Design. Lecture Notes in Computer Science, 146-153. doi:10.1007/978-3-540-73287-7_19

[34] Diehl, J. C., \& Christiaans, H. H. C. M. (2006). Globalization and Cross-Cultural Product Design. International Design Conference - Design 2006 Dubrovnik - Croatia, May 15 - 18, 2006. Retrieved from https://tinyurl.com/v7t8c52

\section{Authors}

Sittisak Rattanaprapawan is a doctoral student in the Architectural Education and Design Program at the Faculty of Industrial Education and Technology at the King Mongkut's Institute of Technology Ladkrabang (KMITL), Bangkok, Thailand. Presently, He is teaching in an undergraduate curriculum in department of industrial product design at Rajamangala University of Technology Isan (RMUTI). He earned a B.S. in Industrial Design. His M.S.I.Ed. (Technology of Industrial Product Design) is also from KMITL. His interests include industrial design, toy design, creative economy, arts, arttoy, character design, and furniture design. He also serves as an adviser to the Ministry of Commerce for OTOP Brands. Email: 57603034@kmitl.ac.th

Apisak Sindhuphak is an Assistant Professor of Industrial Design in the Faculty of Industrial Education and Technology at the King Mongkut's Institute of Technology Ladkrabang (KMITL), Bangkok, Thailand. His research focus includes digital animation, automotive styling, indigenous local materials, mass-customization design processes, biotechnology, and higher-education curriculum design. He has organized course curriculum for the design division and is currently serving as an assistant professor in industrial design for Ministry of Education evaluator. He has published multiple papers in these fields, serves on numerous academic committees for several highly respected journals in Thailand, and conducts design workshop for national universities and around the globe.

Krissana Kiddee is an Assistant Professor in the Faculty of Industrial Education and Technology at the King Mongkut's Institute of Technology Ladkrabang (KMITL), Bangkok, Thailand. Her expertise is in educational research, measurement and evaluation, project evaluation and curriculum development. She is a postgraduate specialist in 
curriculum development and evaluative researches. She has published several papers in these fields, and serves as a reviewer for several highly respected journals in Thailand.

Sompol Dumrongsatien is an Associate Professor in the Faculty of Industrial Education and Technology at the King Mongkut's Institute of Technology Ladkrabang (KMITL), Bangkok, Thailand. His research interests address museum design, the changes in architectural education, vocational education, and work force skills in arts and architecture. He is currently serving as the administrative head for department of Architectural Education and Design, which also consults the area of buildings and Architectural Preservation of Asia Projects.

Article submitted 2020-01-11. Resubmitted 2020-03-12. Final acceptance 2020-03-14. Final version published as submitted by the authors. 